\title{
Does environment affect the star formation histories of early-type galaxies?
}

\author{
I. Ferreras, A. Pasquali, B. Rogers
}

\begin{abstract}
Differences in the stellar populations of galaxies can be used to quantify the effect of environment on the star formation history. We target a sample of earlytype galaxies from the Sloan Digital Sky Survey in two different environmental regimes: close pairs and a general sample where environment is measured by the mass of their host dark matter halo. We apply a blind source separation technique based on principal component analysis, from which we define two parameters that correlate, respectively, with the average stellar age $(\eta)$ and with the presence of recent star formation $(\zeta)$ from the spectral energy distribution of the galaxy. We find that environment leaves a second order imprint on the spectra, whereas local properties - such as internal velocity dispersion - obey a much stronger correlation with the stellar age distribution.
\end{abstract}

\section{The environment of early-type galaxies}

Within the standard framework of structure formation, galaxies grow in a hierarchical fashion from small structures, progressively merging into more massive systems. Galaxies in regions with a higher over-density will collapse earlier than galaxies in under-dense regions. Hence, we expect a significant dependence of the star formation histories of galaxies with the environment were they form and evolve. The morphology-density relation [4] whose 30 year anniversary we celebrate in this Symposium is indeed proof of the fact that environmental mechanisms are important in shaping the galaxy populations we see today. This contribution focuses on the star formation histories of a type of galaxies that are especially sensitive tracers of environment.

The dynamical state of elliptical galaxies suggests a formation process driven by galaxy-galaxy interactions. The current interpretation for their formation history involves major mergers, although an observational quantification of the role of merg-

I. Ferreras

MSSL, University College London, Holmbury St Mary, Dorking, Surrey RH5 6NT, UK. e-mail: ferrerasestar.ucl.ac.uk

A. Pasquali ARI-Universität Heidelberg, Mönchhofstr. 12-14, D-69120 Heidelberg, Germany. e-mail: pasqualieari.uni-heidelberg.de

B. Rogers

Department of Physics, King's College London, Strand, London WC2R 2LS, UK. 
ers and their impact on the underlying stellar populations remains an open question [2, 6]. This morphological type is thus an optimal target to understand the effect of environment on galaxy formation. In order to quantify the effect of environment, we compare large data sets comprising spectral energy distributions of early-type galaxies in different environmental regimes. Differences in their properties are determined by the application of a blind-source separation method whereby the data alone - no modelling - are used to define an observable that discriminates between galaxies based on their spectral information.

The work presented here is based on spectra from the Sloan Digital Sky Survey (SDSS, [12]). By applying Principal Component Analysis (PCA) to the spectra, we find that most of the information locked in the data (over 99\% in the sense of variance) resides in the first two principal components. Projecting all spectra on to a rotated version of these two components allowed us to generate two PCA-based parameters: $\eta$ and $\zeta$. The rotation of components in the parameter space spanned by the eigenvectors of the covariance matrix is often used as a method to go beyond a simple decorrelation of the data, such as independent component analysis, where statistical independence between components is sought (see e.g. [7]). By comparing the rotated components with models of population synthesis for a wide range of star formation histories, we find that the projection of a galaxy spectrum on to the $\eta$ component correlates with average age for metal-rich populations (typical of the types of galaxies explored here). Furthermore, projections on to the $\zeta$ component are very sensitive to the presence of recent star formation, as found when matching against NUV fluxes of early-type galaxies from GALEX (see [5, 8] for details).

Two environmental regimes are studied. In the next section we consider close pairs of interacting early-type galaxies. In $\S 3$ we present the results for a general, volume-limited sample of early-type galaxies out to $\mathrm{z}<0.1$, where environment is parameterised according to the mass of the halo where the galaxy resides, using the groups catalogue from [11].

\section{Environment over small scales: Close pairs}

We define a sample of close pairs by choosing galaxies from SDSS with a radial velocity difference below $500 \mathrm{~km} / \mathrm{s}$ and a physical separation within $30 \mathrm{kpc}$ along the transversal direction. This sample is then visually classified, selecting only those systems consisting of both members being early-type galaxies. Our final sample comprises 347 pairs, and is described in [9]. This definition of a pair produces a clean sample to probe the details of the triggering of star formation and the onset of nuclear emission, as the progenitors are expected to have neither star formation nor AGN activity. The top panels of figure 1 shows the luminosity of the [OIII] line for galaxies with AGN-like emission (which amounts to $9.5 \%$ of the total sample). A trend is found with respect to pair separation (left, given in units of the effective radius of each galaxy), but no correlation is found with internal velocity dispersion (right). The bottom panels show the distribution of the $\zeta$ PCA-component, which 
Ferreras, Pasquali \& Rogers

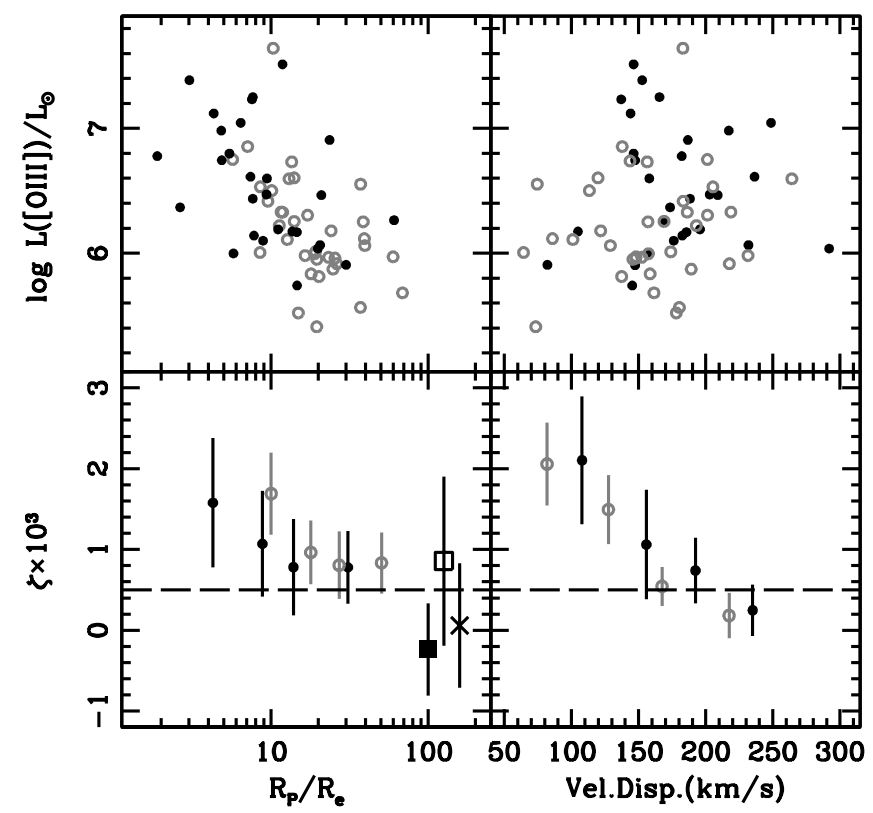

Fig. 1 Spectral properties of the close pairs sample, given by the [OIII] luminosity (top) and PCA parameter $\zeta$, that tracks the underlying stellar populations (bottom). Values of $\zeta>500$ (horizontal dashed line) reflect a significant amount of star formation. The black solid and grey open dots represent galaxies with (without) visual signs of interaction (as seen on the SDSS-DR6 images). The dots in the top panel represent individual galaxies (where AGN-like emission is found), whereas the bottom panels give the average and error on the average for subsamples binned according to pair separation ( $\mathrm{R}_{\mathrm{P}}$, left), given in units of the effective radius) or velocity dispersion (right). The solid and hollow squares in the bottom-left panel shows the average and RMS of a field distribution of NUV faint and bright early-type galaxies, respectively, and the cross shows the average value, regardless of NUV flux.

tracks recent star formation. In this case, the general sample (not only AGN) is shown, with the dots and error bars representing the average and its error on subsamples binned according to separation or velocity dispersion. The decomposition into PCA components is explained in detail in [8]. As a comparison, the squares in the bottom left panel give the values of $\zeta$ for a field sample of early-types separated with respect to their NUV flux - a direct tracer of recent star formation. One can apply a threshold at $\zeta>500$ above which most galaxies will have undergone recent star formation (horizontal dashed line). The comparison shows that early-type galaxies in close pairs have a higher rate of recent star formation than the general sample. The trend with respect to pair separation is visible, although weaker than the correlation found with respect to a more intrinsic observable, namely velocity dispersion. The observed onset of star formation in close pairs, even before the galaxies 


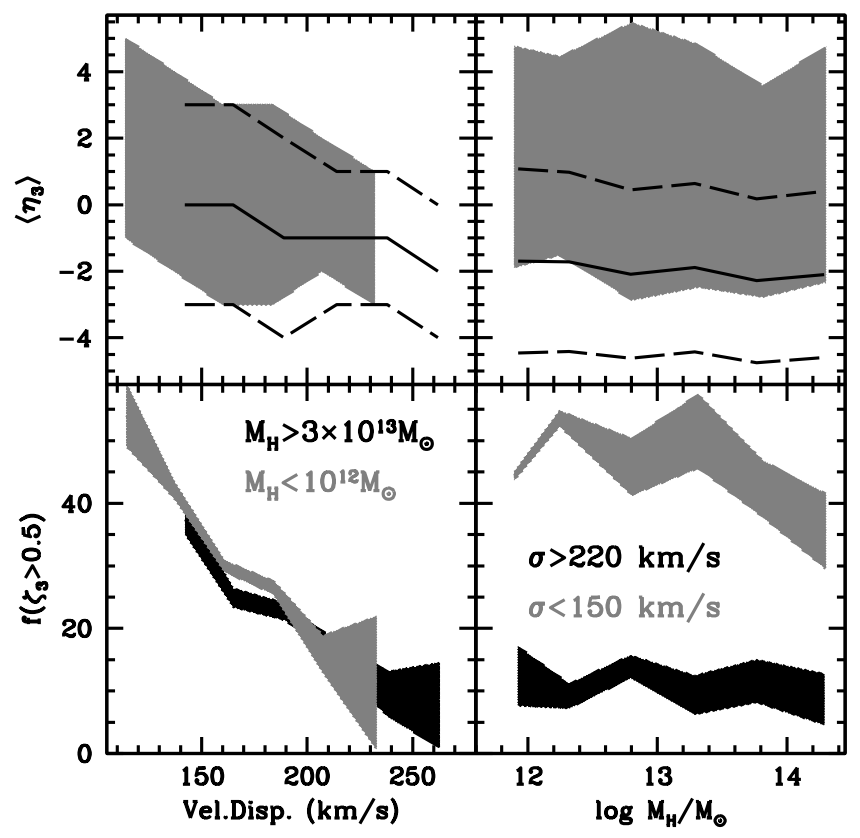

Fig. 2 Effect of group environment on the stellar populations of early-type galaxies. The top panels show the value of PCA parameter $\eta_{3} \equiv \eta \times 10^{3}$, which maps average stellar age. The bottom panels give the fraction of galaxies with recent star formation, as characterized by the PCA parameter $\zeta_{3} \equiv \zeta \times 10^{3}>0.5$. The samples are shown with respect to a local property (velocity dispersion, left) and an environment-related property (group halo mass, right).

display any visual feature of interaction, can be explained by the presence of clouds of gas within their halos, as observed by, e.g.[3]. Even in galaxies separated over 100 effective radii, we find that their level of recent star formation is higher than the average value found for a general sample of (non close pair) early-type galaxies, shown by the ' $x$ ' sign on the bottom-left panel of figure 1 Furthermore, the strong correlation between pair separation and AGN activity - traced by the luminosity of the [OIII] line - suggests that the triggering of star formation precedes AGN activity (see [9] for details)

\section{Environment over large scales: Groups}

For a more general description of environment, we use a sample of SDSS early-type galaxies from [1] and define their environment according to the host dark matter halo, given in the groups catalogue of [11]. This definition of "group" is more gen- 
Ferreras, Pasquali \& Rogers

eral than the traditional concept of galaxy groups, and can extend from isolated galaxies - where one dark matter halo contains only one galaxy, to clusters. However, our sample treats galaxies within a group in the same way, so that we do not resolve, for instance, the environmental differences between the central region and the outskirts of a galaxy cluster. Rather, we want to quantify the importance of the background density where the galaxy lives, on its past and recent star formation history.

Each galaxy is described by a "local" observable (their central velocity dispersion) and by an "environmental" parameter (the host halo mass). Figure 2 shows the trend of the PCA-based parameters ( $\eta$ and $\zeta$, with the subindex ' 3 ' meaning $\times 10^{3}$ ), with respect to the local and environmental observables. The shaded areas map the RMS of the distribution, segregated with respect to halo mass or velocity dispersion, as labelled. For clarity, the black shaded area in the top panels is replaced by solid and dashed black lines, tracing the mean and RMS, respectively. The bottom panel shows the fraction of galaxies with a value of $\zeta$ above the 500 threshold for which the galaxy is assumed to have undergone recent star formation (see the comparison in the bottom left panel of figure 1 between $\zeta$ and NUV bright/faint galaxies). The figure suggests local properties, such as velocity dispersion, play an important role in shaping the underlying stellar populations, both for the average age ( $\eta$, top panels) and for the amount of recent star formation ( $\zeta$, bottom panels). Environment, as defined here by halo mass, only gives a correction to this trend, with galaxies in less massive halos appearing with a similar average age, but with an slightly higher amount of recent star formation at fixed velocity dispersion. We should emphasize here that this definition of environment is adequate for large scale structure analyses. The more acute effects of environment, such as those encountered when galaxies fall into the potential wells of clusters, are not accounted for in this definition.

\section{References}

1. Bernardi, M., Nichol, R. C., Sheth, R. K., Miller, C. J., Brinkmann, J., 2006, AJ, 131, 1288

2. Conselice, C. J., 2009, MNRAS, 399, L16

3. Combes, F., Young, L. M., Bureau, M., 2007, MNRAS, 377, 1795

4. Dressler, A., 1980, ApJ, 236, 351

5. Ferreras I., Pasquali, A., de Carvalho, R. R., de la Rosa, I. G., Lahav, O., 2006, MNRAS, 370, 828

6. Ferreras, I., Lisker, T., Pasquali, A., Khochfar, S., Kaviraj, S., 2009, MNRAS, 396, 1573

7. Hyvärinen, A., Karhunen, J., Oja, E., Independent Component Analysis, 2001, Wiley

8. Rogers, B., Ferreras, I., Lahav, O., Bernardi, M., Kaviraj, S., Yi, S. K., 2007, MNRAS, 382, 750

9. Rogers, B., Ferreras, I., Kaviraj, S., Pasquali, A., Sarzi, M. 2009, MNRAS, 399, 2172

10. Rogers, B., Ferreras, I., Pasquali, A., Bernardi, M., Lahav, O., Kaviraj, S., 2010, MNRAS, 405,329

11. Yang, X., Mo, H. J., van den Bosch, F. C., Pasquali, A., Li, C., Barden, M., 2007, ApJ, 671, 153

12. York, D. G., et al. 2000, AJ, 120, 1579 